\title{
Prospects for chlorophyll fluorescence remote sensing from the Orbiting Carbon Observatory-2
}

\author{
Christian Frankenberg ${ }^{\mathrm{a}, *}$, Chris O'Dell ${ }^{\mathrm{b}}$, Joseph Berry ${ }^{\mathrm{c}}$, Luis Guanter ${ }^{\mathrm{d}}$, Joanna Joiner ${ }^{\mathrm{e}}$, Philipp Köhler ${ }^{\mathrm{d}}$, \\ Randy Pollock ${ }^{\mathrm{a}}$, Thomas E. Taylor ${ }^{\mathrm{b}}$ \\ a Jet Propulsion Laboratory, California Institute of Technology, Pasadena, USA \\ b Colorado State University, Fort Collins, CO, USA \\ c Department of Global Ecology, Carnegie Institution for Science, Stanford, CA, USA \\ d Free University of Berlin, Berlin, Germany \\ e NASA Goddard Space Flight Center, Greenbelt, MD, USA
}

\section{A R T I C L E I N F O}

\section{Article history:}

Received 21 August 2013

Received in revised form 4 February 2014

Accepted 7 February 2014

Available online 12 March 2014

\section{Keywords:}

Chlorophyll fluorescence

GPP

OCO

OCO-2

Atmospheric carbon

\begin{abstract}
A B S T R A C T
The Orbiting Carbon Observatory-2 (OCO-2), scheduled to launch in July 2014, is a NASA mission designed to measure atmospheric $\mathrm{CO}_{2}$. Its main purpose is to allow inversions of net flux estimates of $\mathrm{CO}_{2}$ on regional to continental scales using the total column $\mathrm{CO}_{2}$ retrieved using high-resolution spectra in the $0.76,1.6$, and $2.0 \mu \mathrm{m}$ ranges. Recently, it was shown that solar-induced chlorophyll fluorescence (SIF), a proxy for gross primary production (GPP, carbon uptake through photosynthesis), can be accurately retrieved from space using high spectral resolution radiances in the $750 \mathrm{~nm}$ range from the Japanese GOSAT and European GOME-2 instruments. Here, we use real OCO-2 thermal vacuum test data as well as a full repeat cycle (16 days) of simulated OCO-2 spectra under realistic conditions to evaluate the potential of OCO-2 for retrievals of chlorophyll fluorescence and also its dependence on clouds and aerosols. We find that the single-measurement precision is $0.3-0.5 \mathrm{Wm}^{-2} \mathrm{sr}^{-1} \mu \mathrm{m}^{-1}$ (15-25\% of typical peak values), better than current measurements from space but still difficult to interpret on a single-sounding basis. The most significant advancement will come from smaller ground-pixel sizes and increased measurement frequency, with a 100-fold increase compared to GOSAT (and about 8 times higher than GOME-2). This will largely decrease the need for coarse spatial and temporal averaging in data analysis and pave the way to accurate local studies. We also find that the lack of full global mapping from the OCO-2 only incurs small representativeness errors on regional averages. Eventually, the combination of net ecosystem exchange (NEE) derived from $\mathrm{CO}_{2}$ source/sink inversions and SIF as proxy for GPP from the same satellite will provide a more process-based understanding of the global carbon cycle.
\end{abstract}

(C) 2014 Elsevier Inc. All rights reseved.

\section{Introduction}

Photosynthetic uptake of carbon dioxide by terrestrial vegetation is driven by absorbed photosynthetically active radiation (APAR) in the 400-700 nm wavelength region. The efficiency with which photons are used for actual photosynthesis varies because absorbed energy can also be dissipated into heat (non-photochemical quenching, NPQ). A small fraction is also re-radiated at longer wavelengths $(660-800 \mathrm{~nm})$, which is termed fluorescence and has been widely used in photosynthesis research for decades (e.g. Baker, 2008; Campbell, Middleton, Corp, \& Kim, 2008; Corp, Middleton, McMurtrey, Entcheva Campbell, \& Butcher, 2006; Genty, Briantais, \& Baker, 1989; Krause \& Weis, 1984; Moya et al., 2004). Active pulse-modulated fluorometry allows a direct quantification of photosynthetic efficiency by measuring fluorescence at steady

\footnotetext{
* Corresponding author at: Jet Propulsion Laboratory, California Institute of Technology, Pasadena, USA 4800 Oak Grove Drive, Pasadena, CA 91109, USA.

E-mail address: Christian.Frankenberg@jpl.nasa.gov (C. Frankenberg).
}

state as well as upon illumination with a saturating light pulse. Even though this technique cannot be directly applied from space, steadystate sun-induced chlorophyll fluorescence (SIF) also offers the potential to place constraints on gross primary production (GPP) and thereby gain a more mechanistic understanding of ecosystem carbon exchange (Frankenberg, Fisher et al., 2011).

Recently, Joiner et al. (2011), Frankenberg, Butz, and Toon (2011), Frankenberg, Fisher, et al. (2011), and Guanter et al. (2012) showed that SIF can be retrieved based on in-filling of solar absorption features, so called Fraunhofer lines. The first real retrievals were performed using high-resolution spectra of the oxygen A-band region from the Tanso Fourier Transform Spectrometer onboard of the Japanese GOSAT satellite (Hamazaki, Kaneko, Kuze, \& Kondo, 2005; Kuze, Suto, Nakajima, \& Hamazaki, 2009). Later, Joiner et al. (2012) showed that even moderate spectral resolution can be used to infer SIF using either a very broad Fraunhofer line at $866 \mathrm{~nm}$ (using SCIAMACHY) or applying a larger spectral range from 715 to $780 \mathrm{~nm}$ using GOME-2 (Joiner et al., 2013). In general, there is a trade-off between spectral resolution and spatio- 
temporal sampling. On the one hand, high spectral resolution enables more robust and accurate SIF retrievals but on the other hand it sacrifices spatial sampling. GOSAT retrievals (Frankenberg, Butz, et al., 2011; Frankenberg, Fisher, et al., 2011; Guanter et al., 2012; Joiner et al., 2011, 2012), for instance, are well characterized but cannot provide the spatial mapping of GOME-2. Neither will OCO-2 provide full spatial mapping even though it records about 8 times more spectra than GOME-2. Despite these limitations, GOSAT SIF retrievals have already been proven very useful in the analysis of carbon exchange in the tropical Amazon (Lee et al., 2013; Parazoo et al., 2013).

The Orbiting Carbon Observatory-2 (OCO-2), to be launched in July 2014, can alleviate some of the drawbacks of current SIF observations and combine the advantages of both GOSAT and GOME-2. It will enable SIF retrievals similar to GOSAT but will acquire 24 spectra per second instead of one every $4 \mathrm{~s}$. This almost 100 -fold increase in data density compared to GOSAT is combined with much smaller ground-pixels $\left(1.3 \times 2.25 \mathrm{~km}^{2}\right.$ instead of a circular footprint with $10 \mathrm{~km}$ diameter for GOSAT or $80 \times 40 \mathrm{~km}^{2}$ for GOME-2). As such, it has the potential to largely improve current SIF retrievals as well as bringing together scientific communities focused on top-down net ecosystem exchange inversions based on atmospheric $\mathrm{CO}_{2}$ measurements with those using bottom-up estimates derived from remotely sensed vegetation parameters. Here, we will quantify the potential of OCO-2 using both retrievals based on a synthetic full repeat cycle of OCO-2 data as well as real thermal vacuum test data. The main purpose of this publication is to make potential users aware of datasets for vegetation-specific applications that can be expected from the OCO-2. The primary mission objective of OCO-2 is to measure atmospheric $\mathrm{CO}_{2}$ with the precision and accuracy needed for source/sink inversions on regional scales. Thus, the current user community is composed of atmospheric inversion experts and global carbon cycle modelers. The new fluorescence product might be of interest to different scientific communities and this paper tries to bridge this gap as most previous publications on the OCO-2 focused on $\mathrm{CO}_{2}$ only and scientists might be unaware of the potential of OCO-2 fluorescence retrievals and their applications in photosynthesis research and carbon cycle science. The use of atmospheric $\mathrm{CO}_{2}$ in conjunction with fluorescence has just started (Parazoo et al., 2013) and we anticipate more synergistic applications in the future.

\section{The 0 CO-2 satellite}

The OCO-2 instrument is a 3-channel grating spectrometer scheduled to launch into a sun-synchronous orbit (as part of the A-train) in mid 2014. At $3 \mathrm{~Hz}$ readout rate, it will record high resolution spectra

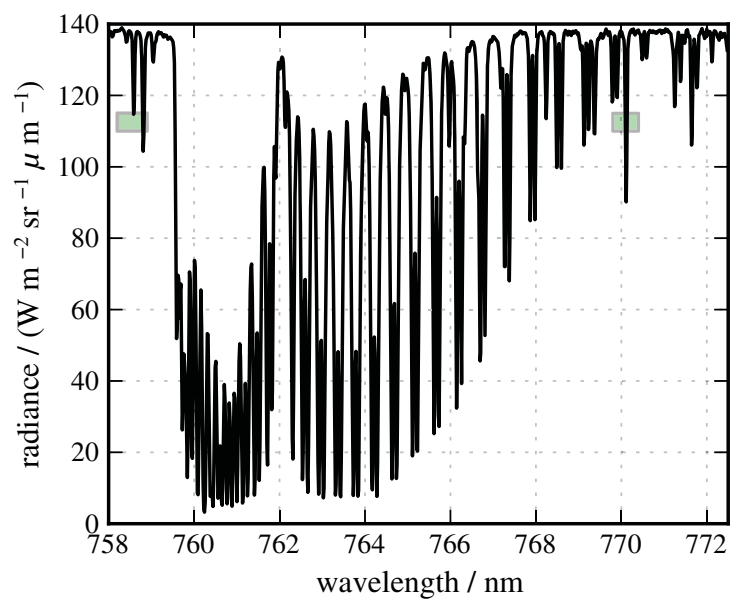

Fig. 1. Exemplary OCO-2 spectrum to be used for chlorophyll fluorescence retrievals. Data were taken during a thermal vacuum test at JPL on April 20, 2012 looking into the sun using a diffuser plate. The primary SIF retrieval fit windows are indicated by transparent green rectangles.

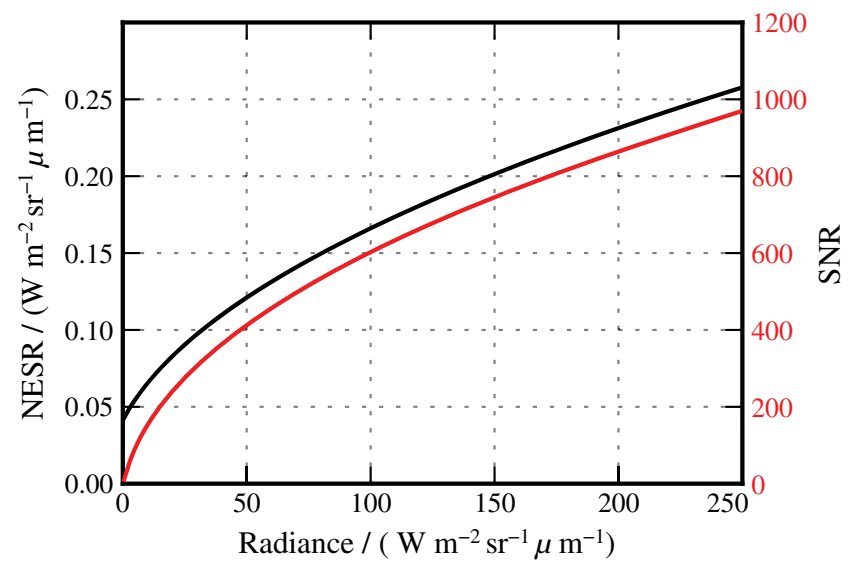

Fig. 2. OCO-2 radiometric performance model in the $\mathrm{O}_{2}-\mathrm{A}$ band. Noise equivalent spectral radiance (NESR) as well as signal-to-noise-ratios (SNR) are shown in black and red, respectively. A Fourier Transform Spectrometer would have a flat spectral response in NESR for a given spectrum (i.e. the NESR at continuum level and in the core of absorption features is identical but a grating spectrometer has lower NESR in the cores compared to the continuum radiance)

of the $\mathrm{O}^{2}$ A-band $(0.757-0.775 \mu \mathrm{m}, \mathrm{FWHM}=0.042 \mathrm{~nm})$, a weak $\mathrm{CO}_{2}$ band $(1.594-1.627 \mu \mathrm{m}, \mathrm{FWHM}=0.076 \mathrm{~nm})$, and a strong $\mathrm{CO}_{2}$ band $(2.043-2.087 \mu \mathrm{m}, \mathrm{FWHM}=0.097 \mathrm{~nm})$ with 8 independent along-slit focal plane array readouts, from here on denoted as footprints. The nominal spatial resolution per footprint is $1.3 \times 2.25 \mathrm{~km}^{2}$ with all 8 individual footprints covering a $10.3 \mathrm{~km}$ full swath width. Fig. 1 shows a real $\mathrm{O}^{2}$-A band example spectrum of the OCO-2 instrument. Similar to the GOSAT, Fraunhofer lines at 758.8 and $770.1 \mathrm{~nm}$ can be used for the fluorescence retrieval.

The current radiometric performance of OCO-2, based on extensive on-ground calibration, is shown in Fig. 2. The noise-equivalentspectral-radiance at zero signal level will correspond to intensities of slightly lower than $0.05 \mathrm{Wm}^{-2} \mathrm{sr}^{-1} \mu \mathrm{m}^{-1}$. In the ideal case of an entirely opaque Fraunhofer line, we could thus measure SIF with a precision of better than $0.05 \mathrm{Wm}^{-2} \mathrm{sr}^{-1} \mu \mathrm{m}^{-1}$ with just a single detector pixel.

In Figs. 1-2, we have displayed full intensities under the assumption of an unpolarized radiance. However, the OCO-2 is only sensitive to intensity with polarization parallel to the slit (Stoke vector components: (I-Q)/2). To maximize signal (esp. in glint mode) and minimize polarization errors, the OCO-2 thus orients its slit perpendicular to the principal plane defined by the sun, the surface footprint, and the instrument aperture.

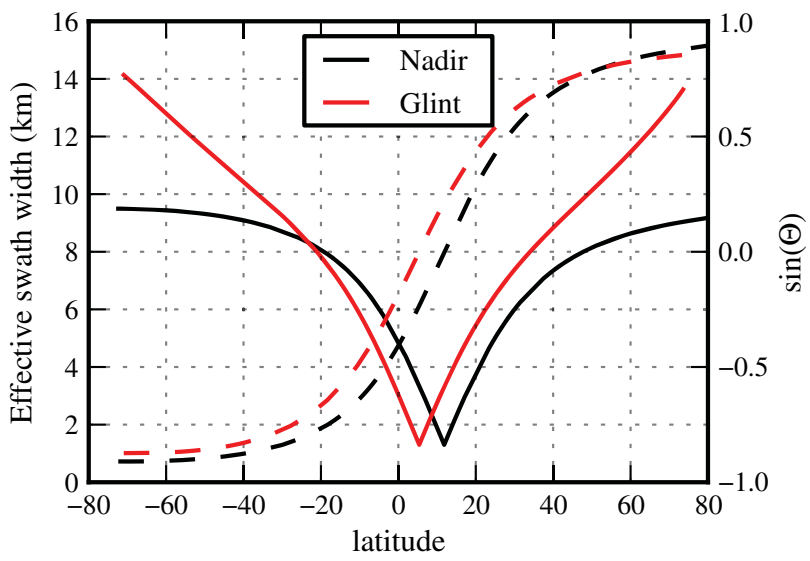

Fig. 3. Typical OCO-2 swath width (lines) and $\sin (\theta)$ (dashed), where $\theta$ is the relative angle between the flight track and slit orientation. Near the equator (depending on season), the slit will be aligned along-track, effectively reducing the effective swath width to the instantaneous field-of-view of a single detector pixel. In glint mode, swaths widths and ground-pixel sizes can be somewhat enlarged owing to the slant viewing geometry. 
OCO2 nadir repeat cycle (16 days)

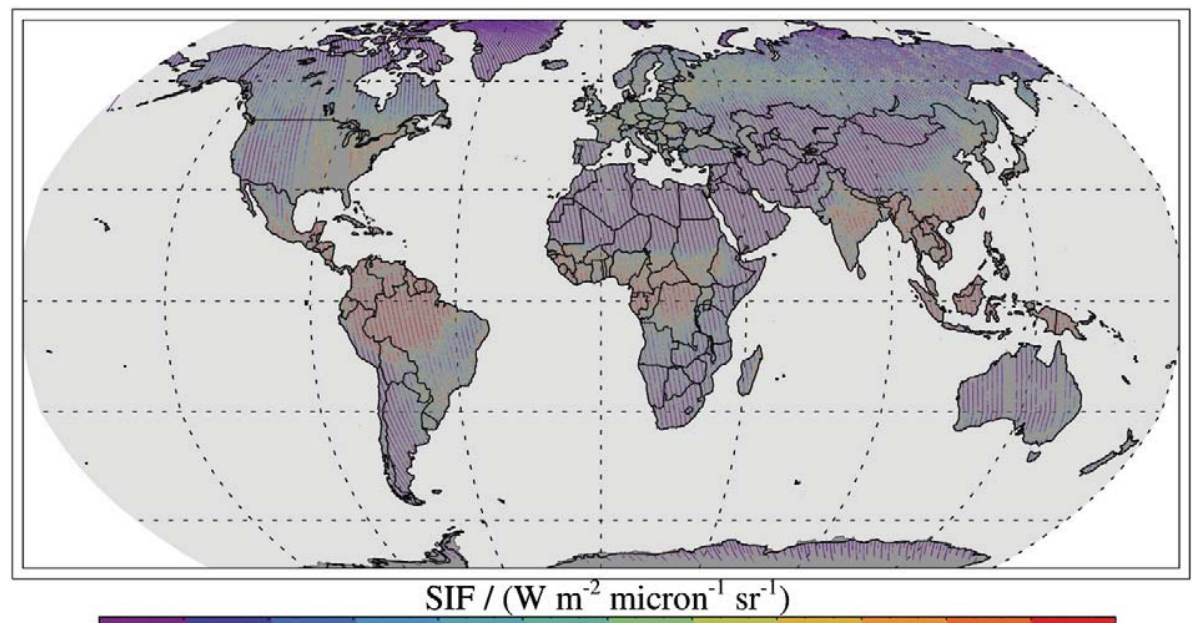

$\begin{array}{lllllllllllll}0.0 & 0.1 & 0.2 & 0.2 & 0.3 & 0.4 & 0.5 & 0.6 & 0.7 & 0.8 & 0.8 & 0.9 & 1.0\end{array}$

Fig. 4. Typical simulated fluorescence retrieval using a 16-day OCO-2 Nadir repeat cycle. The narrow stripes represent a 1.3 to $10.5 \mathrm{~km}$ swath width. Realistic retrieval noise and data yield has been used based on standard cloud-filtering and current OCO-2 signal-to-noise estimates.

This behavior within a typical orbit is depicted in Fig. 3. Slit rotation reduces the effective swath widths, especially near the equator where the slit is aligned along track and OCO-2 footprints basically repeat the same ground-pixel (in which case you will have a very narrow down to $1.3 \mathrm{~km}$ in the extreme case - swath but largely increased sampling within the swath as consecutive footprints will sample the same location during the overflight).

These specific sampling aspects only matter for our specific objectives here and are not that important for atmospheric retrievals where mostly regional-scale spatial gradients in $\mathrm{CO}_{2}$ are targeted. Typical OCO-2 repeat cycles (16-days) will alternate between Nadir (see Fig. 4) and Glint
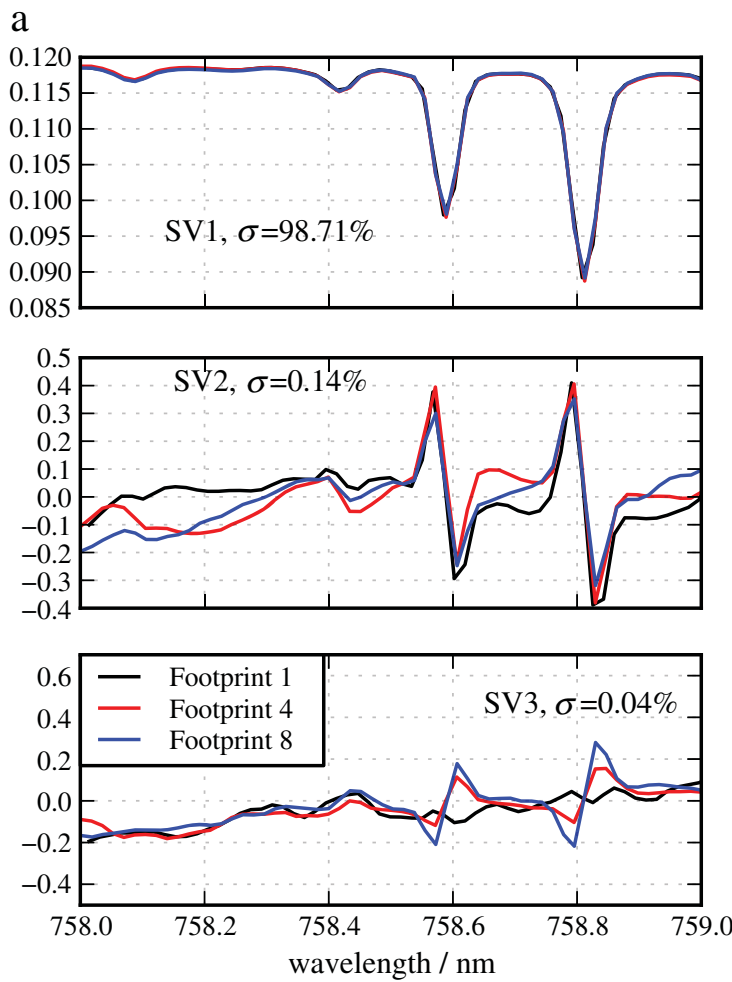

viewing mode and a special target observation mode will be used over selected ground-based validation sites, staring at the site during the overpass and recording thousands of spectra at varying viewing zenith angles. Nadir repeat cycles will always follow exactly the same ground track for each repeat cycle (providing a good dataset for change detection) while the ground tracks in Glint will have somewhat different east-west offsets for each repeat cycle, adding some extended spatial coverage. The changing viewing geometries will have to be taken into account when interpreting SIF data. Nadir spectra of repeating orbits will probably provide the most consistent dataset and might even be useful for the quantification of fluorescence from phytoplankton (Behrenfeld

$\mathrm{b}$
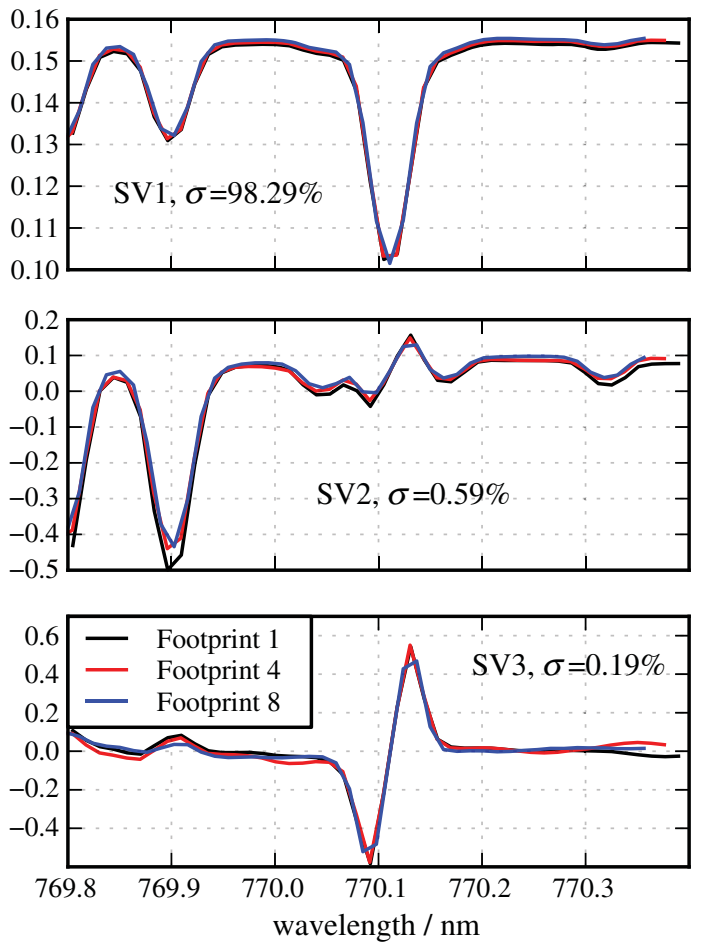

Fig. 5. First three leading singular vectors (SV) of the OCO-2 TVAC test dataset for the two chlorophyll fluorescence retrievals windows covering the Fraunhofer lines at 758.6 and 758.8 nm (left panel) and $770.1 \mathrm{~nm}$ (right panel). The explained variance of each singular value is provided within the respective panels. Only 3 out of the 8 OCO-2 footprints are shown for clarity. 
\& Milligan, 2013). The target mode dataset will enable us to study the bidirectionality of the fluorescence (Van der Tol, Verhoef, \& Rosema, 2009; Van der Tol, Verhoef, Timmermans, Verhoef, \& Su, 2009). Glint observations over land will likely see somewhat lower overall fluorescence values compared to Nadir as the glint spot may partially look at shaded leaves, being opposite to the vegetation hot-spot (Van der Tol, Verhoef, Timmermans, et al., 2009), which is at the same viewing angle but at $0^{\circ}$ azimuth relative to the incident sunlight.

These features specific for the OCO-2 have to be kept in mind when thinking about vegetation-specific remote sensing questions. The OCO2 is not a full mapper like MODIS or GOME-2 because there will be large gaps between orbit paths but sampling along track will be continuous and provide an unprecedented dataset with high accuracy and precision covering very small footprints. We will be able to apply full spectral fitting routines and thus also provide reliable error estimates for retrieved fluorescence data. Along the orbit track, we will also be able to investigate small-scale gradients (on the order of 5-10 km) as a total of about 35 OCO-2 measurements will be recorded while the satellite Nadir point advances by $10 \mathrm{~km}$, reducing the standard error substantially by a factor $1 / \sqrt{n}$, with $n$ being the number of measurements constituting the ensemble average. This way, fine spatial gradients can be properly characterized even within a single orbit path.
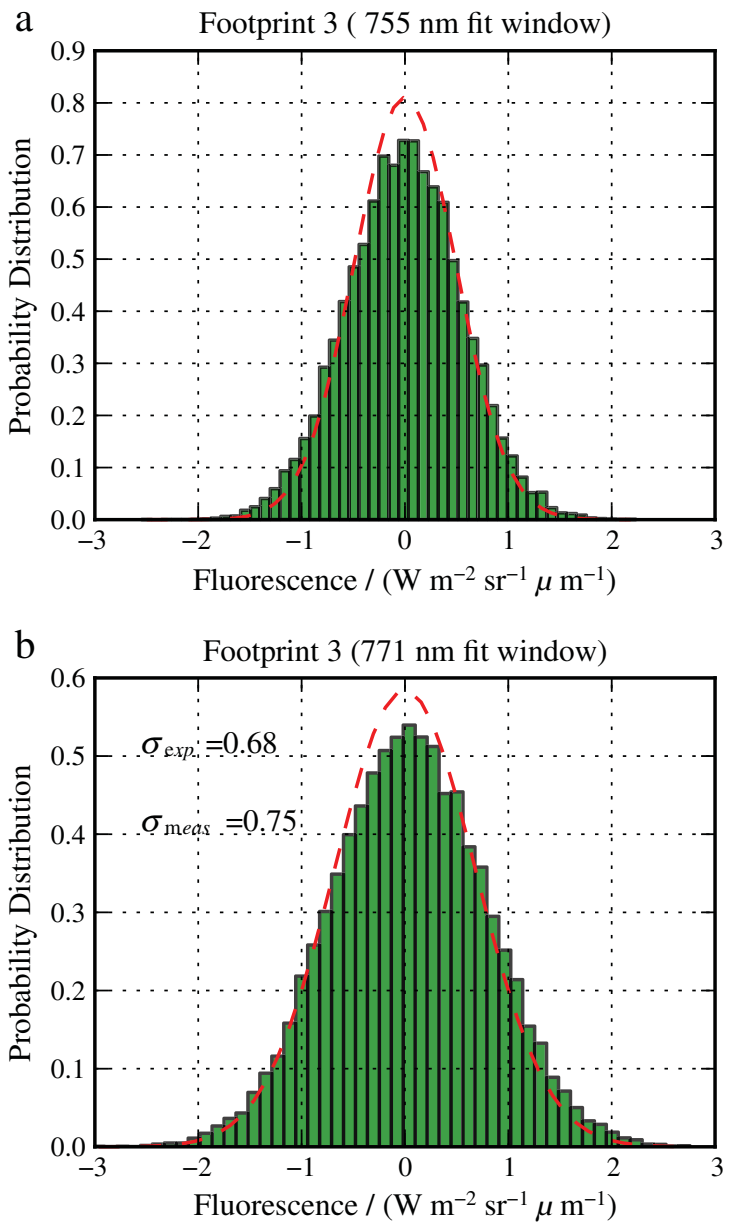

Fig. 6. Histogram of retrieved chlorophyll fluorescence $\left(\mathrm{SIF}_{755}\right)$ from the $758 \mathrm{~nm}$ (top) and $770 \mathrm{~nm}$ (bottom) fit window in one of the OCO-2 footprints. Expected as well as observed standard deviations in retrieved $\mathrm{SIF}_{755}$ are denoted as $\sigma_{\mathrm{exp}}$ and $\sigma_{\mathrm{obs}}$, respectively. The dashed red line represents the expected Gaussian distribution of retrieved SIF based on estimated OCO-2 radiometric performance.

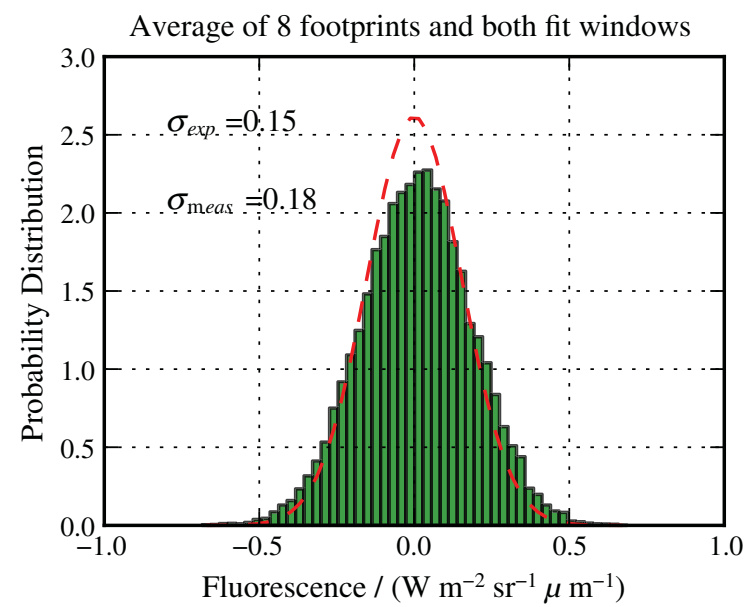

Fig. 7. Histogram of retrieved chlorophyll fluorescence averaged over both fit windows and all 8 footprints. Expected as well as observed standard deviations in retrieved SIF $_{755}$ are denoted as $\sigma_{\mathrm{exp}}$ and $\sigma_{\mathrm{obs}}$, respectively. The dashed red line represents the expected Gaussian distribution of retrieved SIF based on estimated OCO-2 radiometric performance and Gaussian error propagation in the average.

\section{Expected OCO-2 fluorescence retrieval performance}

Retrieval characteristics were evaluated using approximately 80,000 real atmospheric spectra measured during the OCO-2 Thermal VACuum (TVAC) tests at JPL on April 20, 2012. Data were acquired in direct-sun mode, thus no actual chlorophyll fluorescence is apparent in the spectra. However, the inversion procedure for chlorophyll fluorescence can still be performed in order to evaluate expected and observed error characteristics for single soundings as well as accuracy (i.e. checking whether we indeed retrieve zero fluorescence) based on averages over longer time-periods. Here, we use the Singular Value Decomposition (SVD) fluorescence fitting approach (Guanter et al., 2012) as it enables very fast retrievals and also informs us about detector-specific characteristics. As explained in detail by Guanter et al. (2012, 2013), the SVD fitting approach is based on determining singular vectors explaining most of the signal variance in fluorescence-free scenes. Subsequent fitting is performed by linear least squares, retrieving the best linear combination of the singular vectors and a fluorescence-related contribution. We developed a prototype of this methodology for the OCO-2 data and performed the SVD training for all 8 OCO-2 footprints separately as each of them may behave slightly differently (in terms of wavelength calibration, instrument line-shape, dispersion, noise or absolute radiometric calibration).

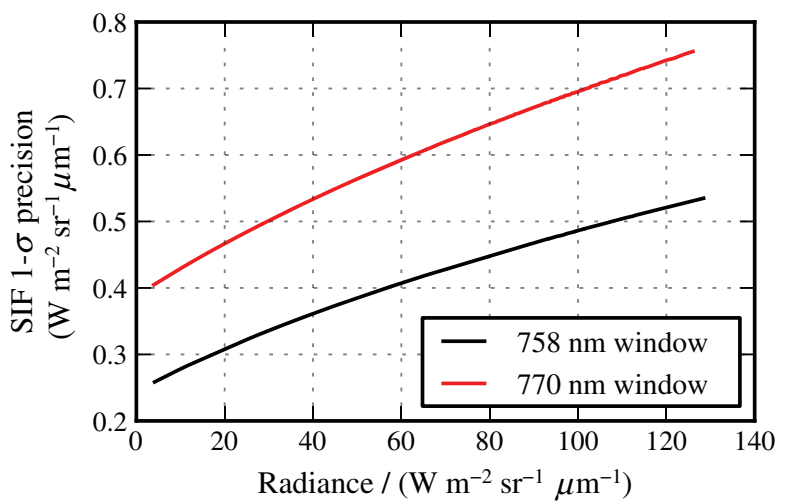

Fig. 8. Single measurement precision error of $\mathrm{OCO}-2 \mathrm{SIF}_{755}$ retrievals in a single footprint in the $758 \mathrm{~nm}$ (black) and $770 \mathrm{~nm}$ (red) spectral windows as a function of continuum radiance level. 


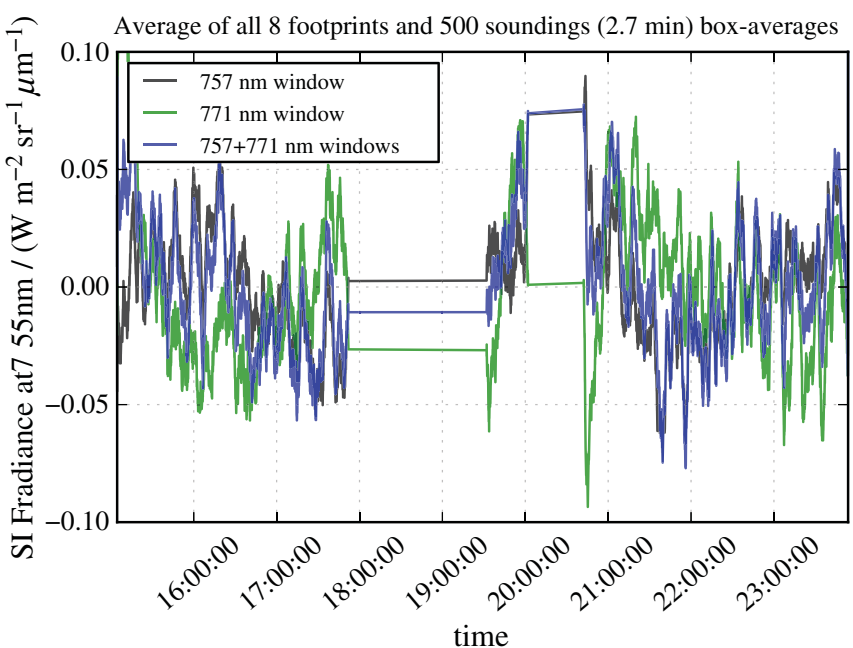

Fig. 9. Smoothed time-series of SIF retrievals using the TVAC data from April 20, 2012 , recorded at JPL. Time is in UTC, straight lines are measurements breaks during which the heliostat was re-aligned.

Fig. 5 shows the leading singular vectors as determined from the OCO-2 Thermal VACuum (TVAC) dataset under a large range of viewing angles. The first singular vector typically represents the main spectral shape and a simple multiplicative factor of this singular vector explains most of the signal variance. In the ideal case, the following singular vectors represent derivatives of the observed radiance with respect to changes in key physical quantities such as Doppler shift, oxygen column amount or viewing geometry. As apparent in Fig. 5, the short wavelength window, which only encompasses the Fraunhofer lines (devoid of oxygen absorption lines) exhibits singular vectors (SV2 and SV3) mostly representing a spectral shift due to the Doppler shifts. Spectral shifts have a non-linear impact on the observed radiance and the singular vectors most likely represent a linear approximation of this effect using two terms. The $770 \mathrm{~nm}$ window includes two stronger oxygen features at 769.8 and $769.9 \mathrm{~nm}$ and the varying optical depths during TVAC (due to varying solar zenith angles) are represented in the second singular vector while the third covers most of the spectral shift effect. The advantage of the SVD approach is that these derivatives are directly computed from the measurements themselves without the need for computationally expensive forward model simulations of the true physics of the process. The non-linear effect of the spectral shift is linearized with the SVD, enabling a simple linear least squares approach for the retrieval. In addition, subtle uncharacterized instrumental features are taken into account at the same time. It is expected that we will perform both the SVD (Guanter et al., 2012) and physical retrievals with spectral fitting fluorescence (Frankenberg, Butz, et al., 2011) for the OCO-2 as computational demand even for the full spectral fitting routine is almost negligible compared to the demand of the full-physics retrieval of the atmospheric $\mathrm{CO}_{2}$ column. The physics-based spectral fitting routine for SIF is based on a simple forward model for the fluorescence emissions and detailed modeling of the instrument response. For OCO-2, each footprint will be characterized with its own dispersion coefficients, instrument line-shape, and noise characteristics. Apart from instrument-specific modifications, the routines are identical to those used for current GOSAT retrievals; see Frankenberg, Butz, et al. (2011) for details. The physics-based spectral fitting routine has the advantage of not being sensitive to the training dataset of the SVD and is also interpretable in terms of its physics-based modeling approach. It may, however, be more susceptible to instrumental features, warranting initial comparisons between the SVD and physics-based approaches once OCO-2 is in orbit.

To assess predicted and true retrieval precision and accuracy, we performed SVD-based fits of chlorophyll fluorescence for the TVAC dataset, where we know that the truth is zero. For both the long and short wavelength fitting window, we define retrieved SIF at $755 \mathrm{~nm}$, assuming a typical spectral shape of SIF. In our case, we use a fixed spectral shape where SIF at $770 \mathrm{~nm}$ is a factor 1.76 lower than at $755 \mathrm{~nm}$ (only a factor 1.1 lower at $758 \mathrm{~nm}$ ), consistent with model estimates (Van der Tol, Verhoef, \& Rosema, 2009) and also GOSAT observations. We follow the retrieval approach by Guanter et al. (2012) and use the first four leading singular vectors in the fitting procedure. The spectrally varying detector noises are taken into account in the weighted least squares approach, also allowing us to directly compute the expected measurement uncertainty matrix.

\subsection{Precision estimates}

Fig. 6 shows histograms of retrieved $\operatorname{SIF}_{755}$ for a representative footprint in the 758 and $771 \mathrm{~nm}$ fitting windows. The expected distribution based on the current OCO-2 detector noise estimates are shown as a red dashed line. One can see that expected and observed

OCO2 nadir repeat cycle (16 days), dp $<100 \mathrm{hPa}$

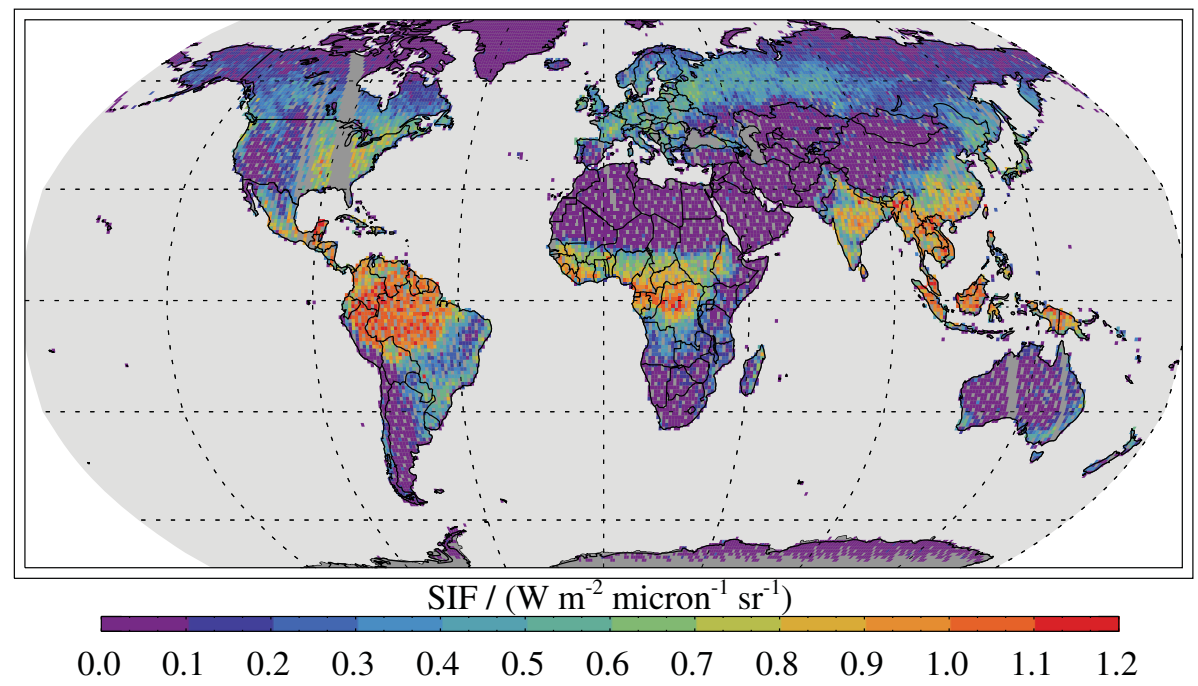

Fig. 10. Typical simulated fluorescence retrieval using a 16-day OCO-2 Nadir repeat cycle, same as Fig. 4 but gridded at $1 \times 1^{\circ}$ and using simple cloud filter, where retrieved surface pressure is within $\pm 100 \mathrm{hPa}$ compared to the meteorological forecast. 
standard deviations differ only by a very small amount but agree to within $10-15 \%$. In this particular case, single measurement precision would be about $0.5 \mathrm{Wm}^{-2} \mathrm{sr}^{-1} \mu \mathrm{m}^{-1}$ in the $758 \mathrm{~nm}$ window and $0.7 \mathrm{Wm}^{-2} \mathrm{sr}^{-1} \mu \mathrm{m}^{-1}$ in the $770 \mathrm{~nm}$ window. Typical real SIF $_{755}$ values observed from GOSAT vary between 0 and $2 \mathrm{Wm}^{-2} \mathrm{sr}^{-1} \mu \mathrm{m}^{-1}$ across the globe, meaning that a single measurement from the OCO-2 will in most cases be too noisy to be useful. However, given the much smaller OCO-2 footprint size, more extreme values in the SIF might be expected so that we don't yet fully rule out the potential of using singlesoundings in case studies.

To fully exploit the potential of OCO-2 on the global scale, measurements will have to be averaged to reduce the standard error in the mean. Pure un-correlated precision errors in the mean of $n$ samples will get reduced by a factor of $1 / \sqrt{n}$.

In Fig. 7, we averaged the SIF retrievals of all 8 footprints as well as the two different fit windows and show histograms of retrieved SIF as
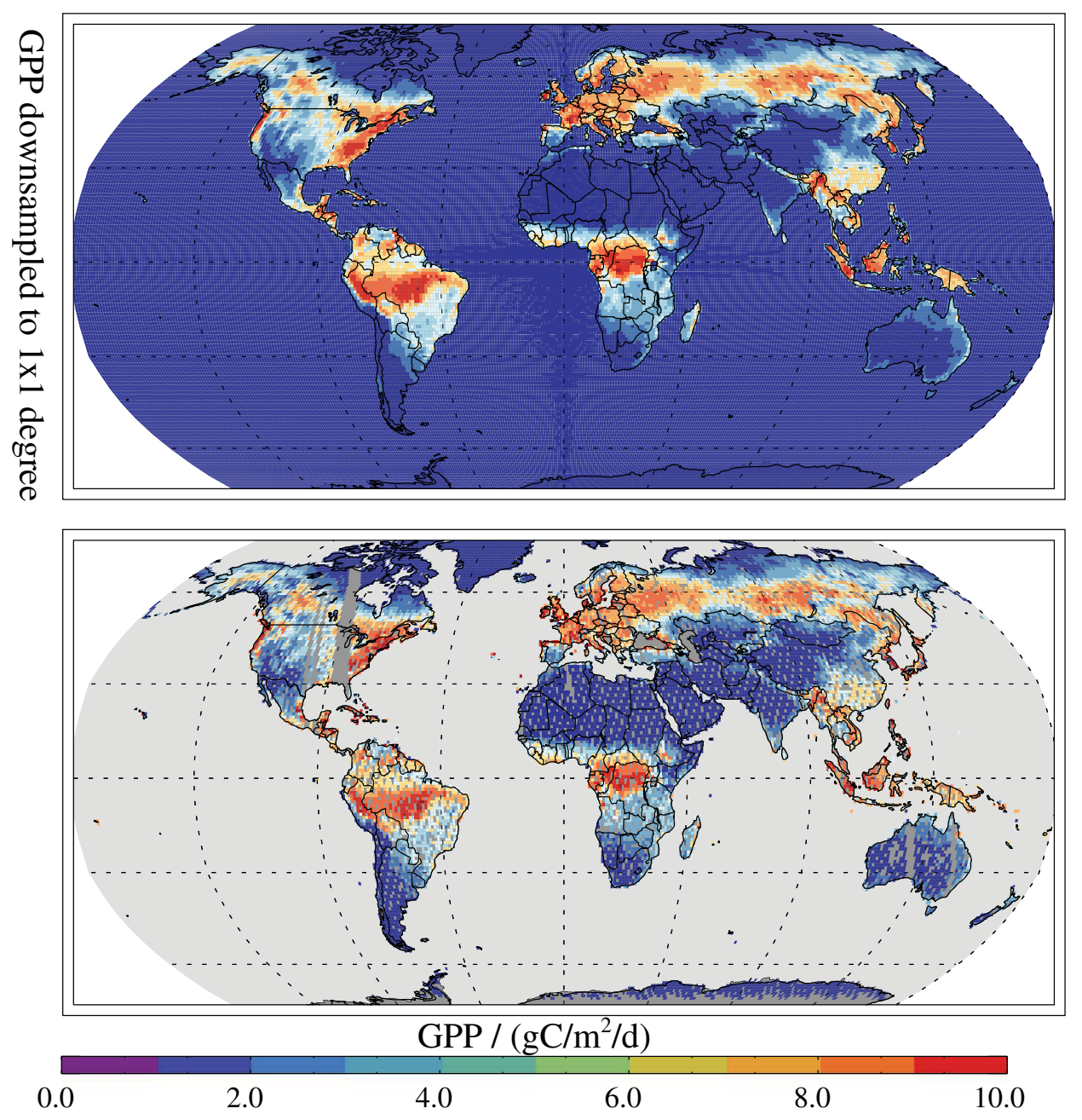

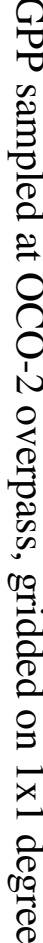

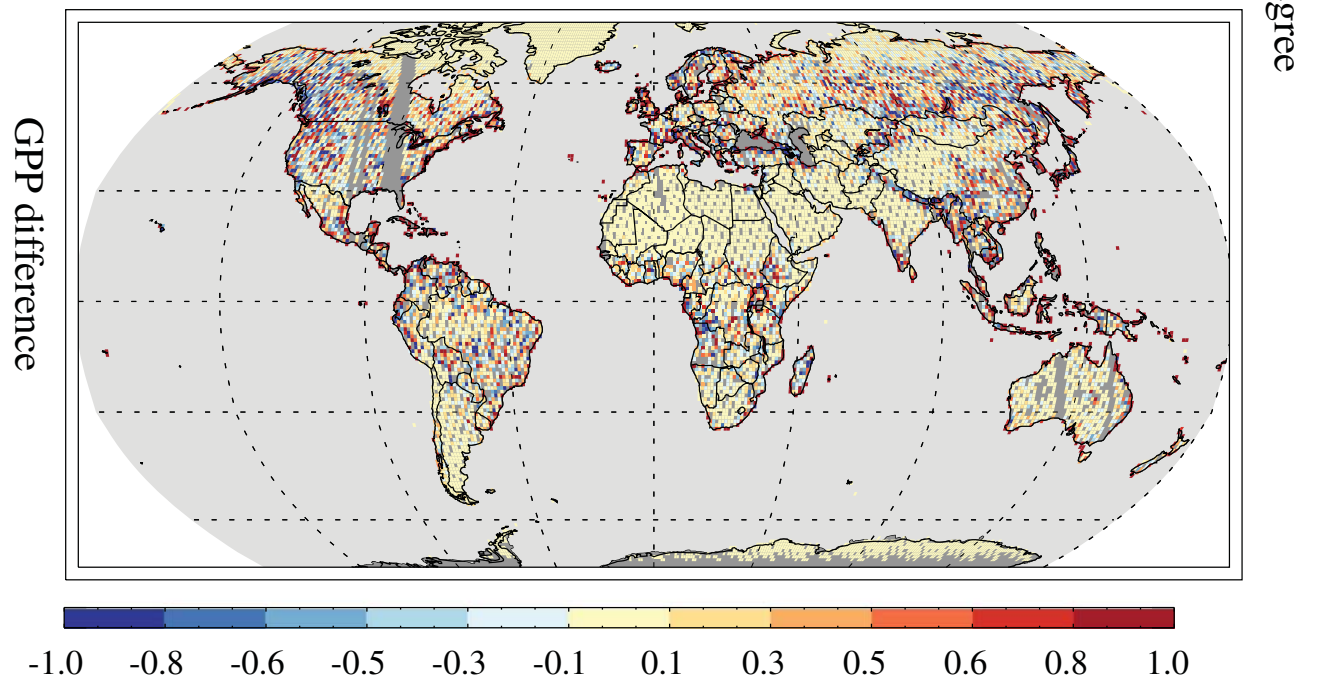

Fig. 11. Sampling bias incurred by OCO- 2 due to incomplete mapping of $1 \times 1^{\circ}$ grid boxes. 
well as expected standard deviations. As expected, the standard error is now largely reduced (to about $0.18 \mathrm{Wm}^{-2} \mathrm{sr}^{-1} \mu \mathrm{m}^{-1}$ ) and retrieved values still appear very Gaussian with an observed standard deviation closely matching the expected single measurement precision error. These averaged SIF values now exhibit a noise level low enough to be used for scientific interpretation on a scale of $10.6 \times 2 \mathrm{~km}$. Different averaging schemes can be envisioned but the main conclusion remains: the vast amount of OCO-2 spectra will allow the SIF retrievals with much higher precision than that of the GOSAT over smaller spatial extents, especially along the track direction.

Given that the SIF retrievals using real atmospheric test data behaved very much as expected, we can use retrieval error simulations to estimate single-measurement precisions expected for real OCO-2 spectra in the orbit.

Fig. 8 shows the single-measurement precision expected for a single OCO-2 footprint as a function of continuum level radiances expected in a typical Nadir orbit. Typical errors range from 0.3 to $0.5 \mathrm{Wm}^{-2} \mathrm{sr}^{-1} \mu \mathrm{m}^{-1}$. A few aspects are noteworthy. First, the TVAC data have been recorded at a relatively high signal level as direct sunlight via a diffusor was observed while the typical range of Nadir signal levels is $10-130 \mathrm{Wm}^{-2} \mathrm{sr}^{-1} \mu \mathrm{m}^{-1}$. TVAC precision errors are thus somewhat higher than what we expect in the orbit. The higher dynamic range in expected OCO-2 radiances is mainly driven by high radiances in glint viewing geometries. Second, the single measurement precision in absolute SIF improves with decreasing signal level. At first glance this may be somewhat counterintuitive as detector signal-to-noise ratios (SNR) and 1- $\sigma$ errors are anti-correlated. The error in the relative contribution of SIF to the overall radiance would indeed increase at lower signal levels. The continuum level baseline is very well characterized with high-resolution spectra as a multitude of spectral points are averaged to determine the baseline. The absolute noise within the Fraunhofer lines is then proportional to the error in SIF and as the absolute noise decreases with signal level, SIF retrievals get more precise. Overall, single measurement precision will be better than for GOSAT as the SNR will rarely drop below 200 and is $200-700$ in most cases (while darker spectra in GOSAT have SNR $<100$ ).

The behavior of the single measurement precision with signal level may also have important implications for ocean retrievals. In Nadir (as observed also over the ocean during the Nadir repeat cycle), background radiances are very small in clear skies, so that SIF can contribute a substantial fraction to the observed radiance. Over-ocean fluorescence originates from photosynthesizing phytoplankton, and is roughly proportional to chlorophyll-a concentration near the ocean surface (Neville \& Gower, 1977). The highest SIF values over the oceans are expected along with the coastlines, which could not be well sampled with GOSAT's observation pattern. The continuous swath of OCO-2 will facilitate these studies. Even though we expect SIF absolute values to be in general smaller for the ocean (compared to land), we believe that the SIF retrievals over the oceans with the OCO-2 might be feasible and complement current MODIS- and MERIS-based fluorescence estimates (Xing et al., 2007). Unfortunately, it bears noting that ocean water absorbs light about 6 times more strongly near OCO-2 fluorescence wavelengths of $\approx 760 \mathrm{~nm}$ compared to the MODIS observation wavelength of $677 \mathrm{~nm}$. Therefore, while MODIS observations are sensitive to the chlorophyll fluorescence in roughly the top two meters of the ocean, OCO-2 will only be sensitive to fluorescence originating in the top half-meter or less of the ocean. In addition, the fluorescence peak at $740 \mathrm{~nm}$ is much lower than the one at $680 \mathrm{~nm}$, which is used for MODIS fluorescence retrievals and ocean Raman scattering may also play a role. Given the low computational demand of the SIF algorithm, retrievals will be performed in any case over all surface types, also over the ocean. However, as stated above, we cannot yet confidently assert that OCO-2 SIF retrievals over the oceans will be scientifically useful.

\subsection{Accuracy estimates}

The question arises as to which level the assumption of un-correlated and purely statistical retrieval noise holds and which accuracy levels one might expect from the OCO-2. To test this, we used retrievals of the entire TVAC dataset and computed a smoothed time-series of retrieved SIF.

Fig. 9 shows the corresponding plot, independently for the two fit windows as well as combined. The precision error in these cell averages is on the order of $0.01 \mathrm{Wm}^{-2} \mathrm{sr}^{-1} \mu \mathrm{m}^{-1}$ but significant deviations from 0 can be observed, up to $0.05 \mathrm{Wm}^{-2} \mathrm{sr}^{-1} \mu \mathrm{m}^{-1}$. There is also a high frequency (about $15 \mathrm{~min}$ ) oscillatory pattern in the retrieved SIF, which may be related to a cyclical periodicity in the solar tracking system. In addition, the OCO-2 slit may not have been fully illuminated during the TVAC. While it shows that very subtle instrument features can cause systematic biases of up to $0.05 \mathrm{Wm}^{-2} \mathrm{sr}^{-1} \mu \mathrm{m}^{-1}$, we are confident that this can be seen as an upper bound for in-orbit performance where both the instrument as well as slit illumination are better controlled. However, we will re-assess SIF retrieval accuracy on-orbit using statistics over non-fluorescent targets with variable signal levels such as deserts and ice or footprints at surface temperatures well

OCO2 nadir repeat cycle (16 days), no filter

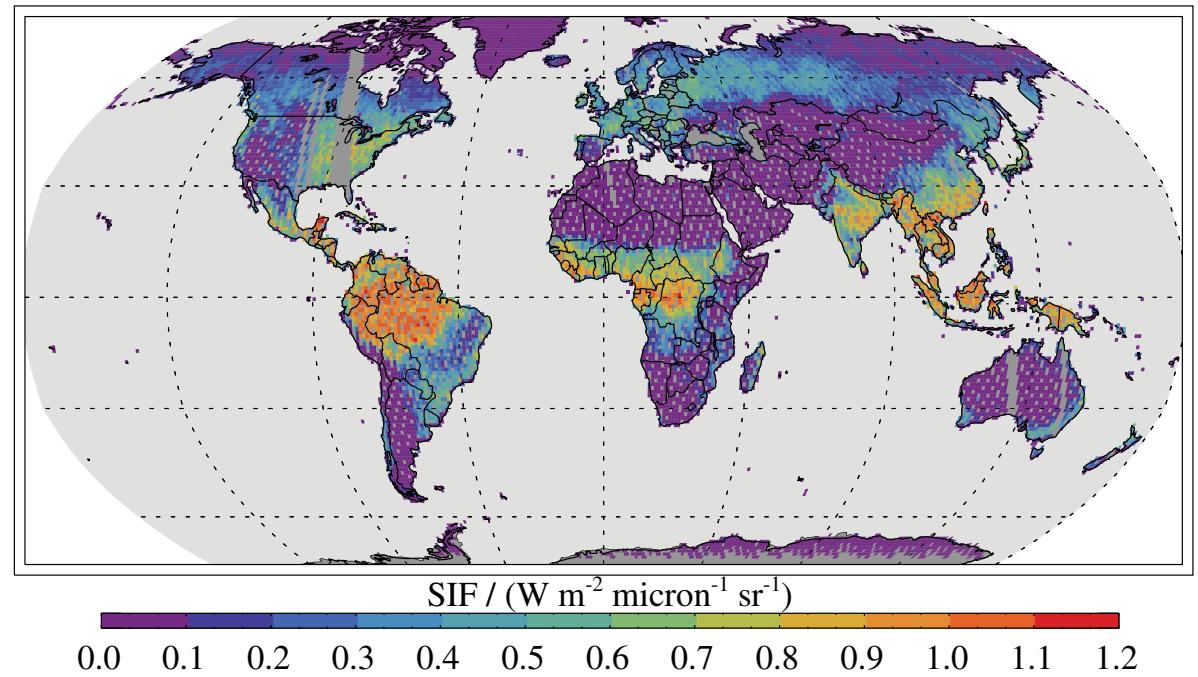

Fig. 12. SIF retrievals of an entire OCO-2 repeat cycle without application of any cloud filter. Visually, it is almost indistinguishable from Fig. 10. 
a OCO2 nadir repeat cycle (16 days), no filter

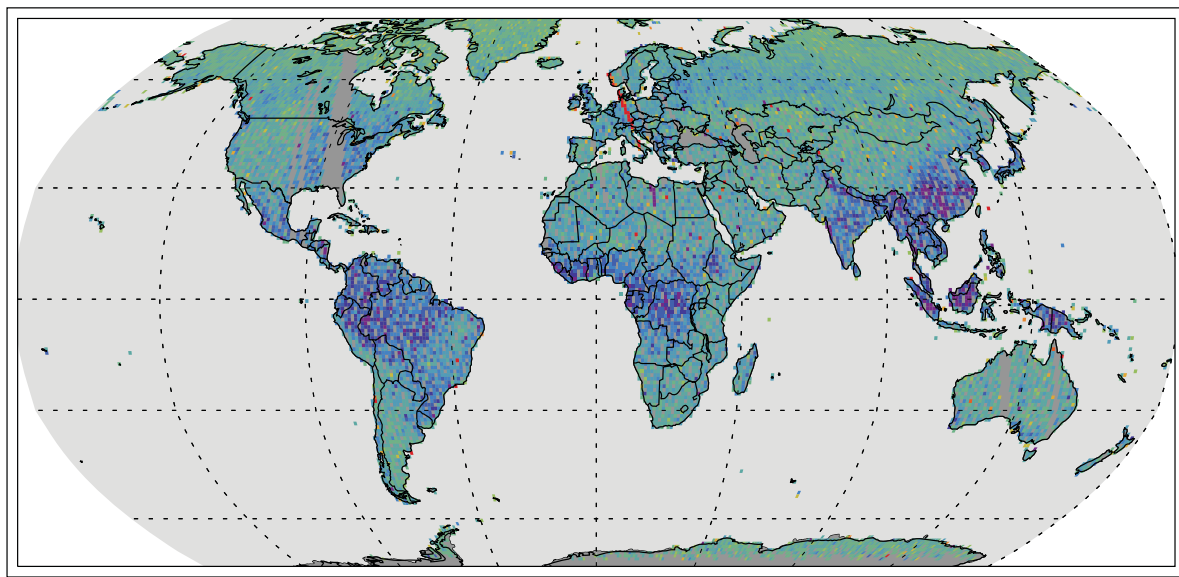
delta-SIF / $\left(\mathrm{W} \mathrm{m}^{-2}\right.$ micron $\left.^{-1} \mathrm{sr}^{-1}\right)$

$\begin{array}{lllllllllllll}-0.30 & -0.25 & -0.20 & -0.15 & -0.10 & -0.05 & 0.00 & 0.05 & 0.10 & 0.15 & 0.20 & 0.25 & 0.30\end{array}$

b $\quad 0$ CO2 nadir repeat cycle (16 days), dp $<100 \mathrm{hPa}$ filter

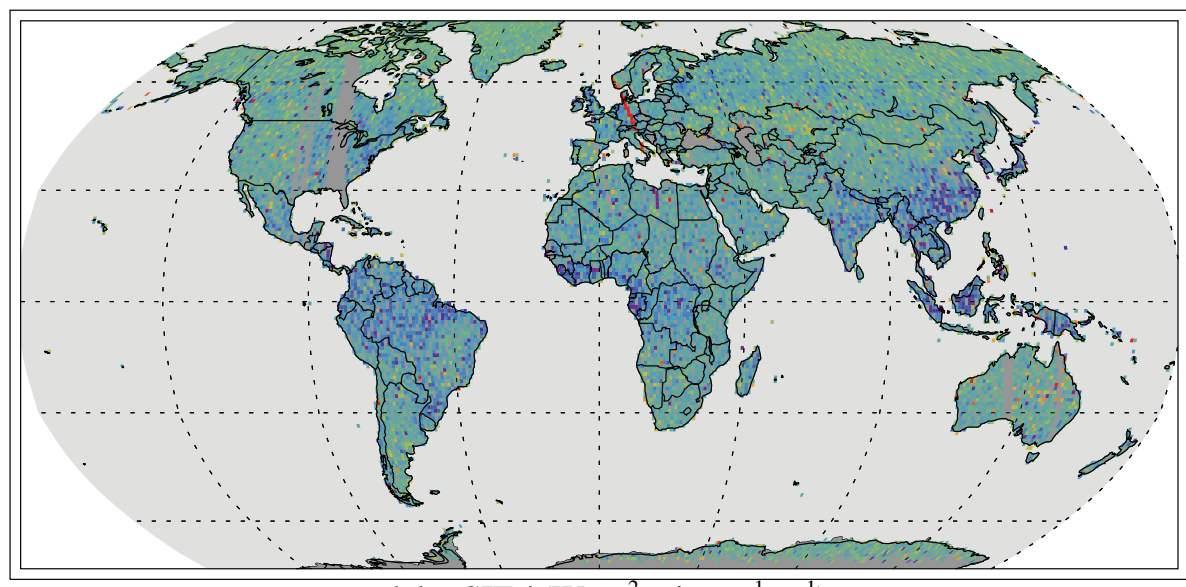

delta-SIF / $\left(\mathrm{W} \mathrm{m}^{-2}\right.$ micron $\left.^{-1} \mathrm{sr}^{-1}\right)$

$\begin{array}{lllllllllllll}-0.30 & -0.25 & -0.20 & -0.15 & -0.10 & -0.05 & 0.00 & 0.05 & 0.10 & 0.15 & 0.20 & 0.25 & 0.30\end{array}$

c $\quad 0$ OCO2 nadir repeat cycle (16 days), dp $<50 \mathrm{hPa}$ filter

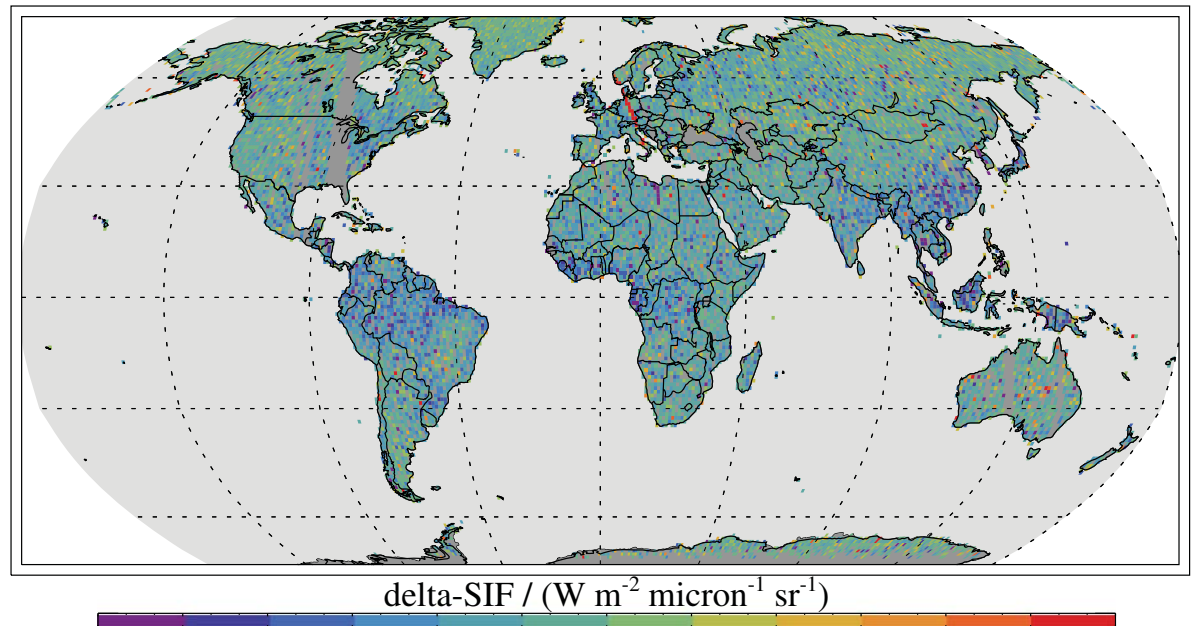

$\begin{array}{lllllllllllll}-0.30 & -0.25 & -0.20 & -0.15 & -0.10 & -0.05 & 0.00 & 0.05 & 0.10 & 0.15 & 0.20 & 0.25 & 0.30\end{array}$ 
d $\quad$ OCO2 nadir repeat cycle (16 days), dp<10hPa filter

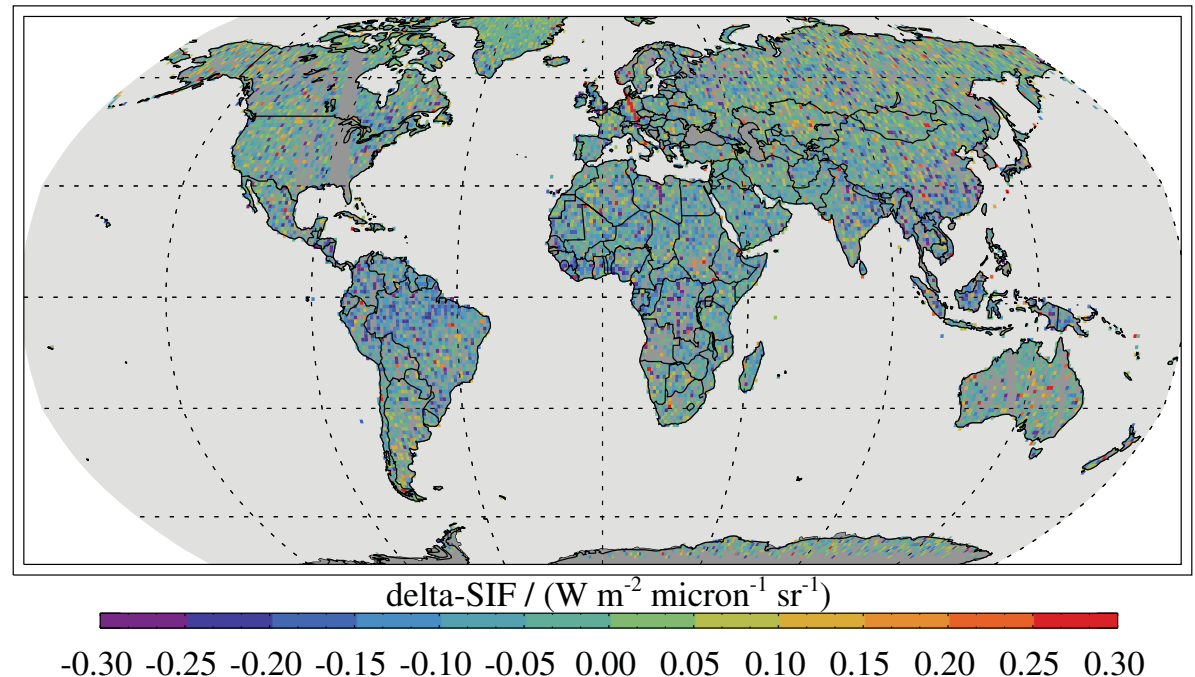

Fig. 13. Absolute biases in retrieved SIF as a function of a surface-pressure retrieval based cloud-filtering scheme (Taylor et al., 2012). The threshold (dp) is based on the absolute difference between the a priori surface pressure from meteorological forecast and the retrieved surface pressure using the entire $\mathrm{O}_{2}-\mathrm{A}$ band.

below freezing. In summary, we have shown that most of the noise in the retrieved SIF can be readily reduced by averaging and follows the $1 / \sqrt{n}$ law. However, analysis of variations on the order of only $0.02-0.05 \mathrm{Wm}^{-2} \mathrm{sr}^{-1} \mu \mathrm{m}^{-1}$ should be handled with care as accuracy errors will become significant. Assuming the empirical linear relation between SIF and GPP as observed by Frankenberg, Fisher, et al. (2011), the above values correspond to GPP values of only $0.13-0.32 \mathrm{~g} \mathrm{~cm}$ -2 day $^{-1}$.

\section{Expected OCO-2 fluorescence datasets on the global scale}

So far, we have only analyzed the expected OCO-2 performance on a single-sounding or ensemble averages. In this section, we will cover aspects relevant to carbon cycle modelers on the global scale, i.e.

- What impact does the footprint size have on SIF retrievals?

- What errors on the regional scale are introduced by preferential and incomplete sampling of OCO-2 only along a narrow orbit track?

- What datasets can I expect within a typical OCO-2 repeat cycle, how many days of averaging do I need at what grid-scale?

- How do clouds and aerosols affect the SIF retrievals given potentially imperfect filtering? What is the trade-off between cloud-filtering, accuracy and data yield?

\subsection{Impact of footprint size}

A major concern of the community working with remotely sensed vegetation datasets is the footprint size. This is due to two factors: 1) Local studies are interested in very specific and potentially smallscale features (e.g. agricultural plots) and 2) ground pixels should ideally be homogenous as sub-pixel heterogeneity can bias the retrieval of vegetation indices. For example, a footprint which is half covered with snow and half with vegetation won't have the same NDVI as the average of the two individual NDVI of snow and vegetation. Due to nonlinearities, the averages of retrieved quantities based on individual radiances $I i$ and the retrieved quantity based on averaged radiances are not the same:

$\sum_{i} \operatorname{NDVI}\left(I_{i}\right) \neq \operatorname{NDVI}\left(\sum_{i} I_{i}\right)$
Owing to this inequality, footprints have to be homogenous to be unbiased for classical optical remote sensing of vegetation indices. SIF, however, is different in its retrieval characteristics as it is only sensitive to emissions emanating from the surface, with non-fluorescing targets (snow, streets, etc.) not contributing to the signal. We essentially measure the emitted radiance integrated over the field-of-view and as such, the operators for the SIF retrievals are by definition interchangeable:

$\int_{\text {FOV }} \operatorname{SIF}(I)=\operatorname{SIF}\left(\int_{\text {FOV }} I\right)$

In the extreme case, we could thus argue that the measurement of the (day-lit) hemispherically integrated SIF from the Earth can provide a proxy for the global gross primary production (under the assumption of a linear relation, as empirically found by Frankenberg, Fisher, et al. (2011)). However, spatial context will of course be lost but this extreme example illustrates that spatial resolution for fluorescence retrievals is irrelevant unless you want to attribute specific patterns to specific biomes or local processes. We basically measure the spatially integrated photon-flux of a by-product of photosynthesis and the spatial resolution depends on the interest of the user community and is not a prerequisite in itself. However, OCO-2 will push the spatial resolution of SIF measurements to a new level, allowing more detailed and local researches than is currently possible. But we should also keep in mind that most global vegetation modelers - not to speak of inverse modelers using atmospheric $\mathrm{CO}_{2}$ data - don't work with high resolution imagery but only with down-scaled vegetation index products.

4.2. Impact of preferential sampling along the narrow OCO-2 swath, what coverage and data to expect

To answer these questions, we analyzed a full repeat cycle (16 days) of simulated OCO-2 data for September. We used the OCO-2 orbit simulator (see Appendix A), which now includes a fluorescence signal that is geographically distributed by linearly scaling $0.5^{\circ}$ resolution GPP maps from Beer et al. (2010). The simulated data include realistic cloud and aerosols fields based on CALIPSO statistics (Winker, Hunt, \& McGill, 2007) and also include full multiple scattering radiative forward modeling of the fluorescence emission emanating from the surface. Details of the simulations can be found in the Appendix A. As can be seen in 
Fig. 4, the OCO-2 only samples a very narrow swath with large gaps inbetween orbit paths.

In Fig. 10 , we use exactly the same data but grid them at $1 \times 1^{\circ}$. This results in a quasi-global coverage albeit with sampling errors associated with each of the larger grid cells. To quantify the sampling bias in this potential high-level product and its relevance for regional scale carbon cycle modeling, we analyzed high-resolution MODIS GPP data $\left(0.05^{\circ}\right)$ and its dependence on preferential sampling at the $1 \times 1^{\circ}$ grid. The top panel of Fig. 11 shows the high-resolution MODIS GPP dataset regridded at $1 \times 1^{\circ}$ based on the full dataset. The middle panel shows the grid cell averages based on samples just along with the direct Nadir point of the OCO-2 instrument, i.e. with a very limited though stochastic sampling. The difference between the two is displayed in the lower panel, showing that overall only relatively small and spatially uncorrelated errors exist. The largest differences are observed along coastlines, where grid cells cross the land/ocean boundary and we averaged OCO-2-based data only over land pixels while the high resolution MODIS GPP was averaged over all pixels. Overall though errors are small in most places, showing that despite the narrow swath, OCO-2 data aggregated to larger scales (e.g. $1 \times 1^{\circ}$ ) are representative of true grid cell averages. Even the small errors can be avoided if OCO-2 fluorescence data is gridded at finer spatial resolution, albeit at the expense of apparent coverage (gaps in the maps). For visualization, gap-free global maps will be preferred but detailed analysis can take the spatial sampling fully into account.

It is worth mentioning that OCO-2 will collect as many spectra within one repeat cycle as the GOSAT does in slightly more than 4 years. This was the main barrier of using GOSAT fluorescence retrievals at shorter temporal and spatial scales because high single-measurement noise in conjunction with low data amount resulted in high standard errors and the subsequent need for large-scale averaging to suppress the apparent noise. The effect of noise is already included in the OCO-2 simulated retrievals in Fig. 10, showing that the OCO-2 will be able to provide smooth global distributions of SIF bi-weekly.

\subsection{Impact of atmospheric scattering on SIF retrievals: curse or blessing?}

We have shown in Frankenberg, O'Dell, Guanter, and McDuffie (2012) that SIF retrievals (if based on Fraunhofer lines) are very insensitive to atmospheric scattering, with more than $80 \%$ of the emanating fluorescence at the canopy scale reaching the detector in space even up to optical densities of 4 (as can be seen in Fig. 12, which didn't include any cloud filtering at all). Unlike reflectance-based products, the SIF retrievals are mostly subdued by atmospheric scattering, mostly when optical densities are very high and/or single scattering albedo low. In principle, these highly scattering scenes can be easily filtered out, especially because the operational OCO-2 preprocessing filters (O'Dell et al., 2012) for atmospheric $\mathrm{CO}_{2}$ retrievals are very strict and only pass very clear scenes $(\approx<0.3 \mathrm{OD})$. This could minimize a potential systematic low-bias in the SIF. The question is, is this desirable and does it reflect the true variability in GPP? Aren't we trading accuracy in the SIF with a clear-sky bias in our observations? As absorbed photosynthetic active radiation (APAR) is reduced in highly scattering scenes, the true SIF (and GPP) will also be subdued. If we can quantify the signal loss in the SIF from the canopy to the top-of-atmosphere (TOA), we could thus truly capture changes even under cloudy conditions and thereby minimize a clear-sky bias in SIF observations. Further studies, especially in regard to changes in fluorescence and photosynthesis yield under changing illuminations, will be needed to quantify this effect. This will pave the way towards the quantification of photosynthesis and its efficiency in atmospheric scattering conditions, potentially even looking at GPP enhancements in scattered light conditions, which has a large control on GPP (Gu et al., 2002, 2003; Kanniah, Beringer, North, \& Hutley, 2012). However, we acknowledge that more studies are needed towards that long-term goal.
Fig. 13 shows the bias in the retrieved SIF depending on the choice of a cloud-filter threshold. The bias is defined as the difference between retrieved top-of-atmosphere and top-of-canopy SIF. The cloud-filtering scheme is part of the operational OCO-2 pre-processing pipeline. The effect is in general largest in the tropics with the most persistent cloudcover. One can see that a relatively relaxed threshold of $100 \mathrm{hPa}$ already largely reduces the biases compared to the no-filtering case while strict cloud-filtering ( $10 \mathrm{hPa}$ threshold) minimizes biases but leads to a more noisy map as the total number of measurements used is reduced. We propose that a filter threshold of $50-100 \mathrm{hPa}$ should be sufficient for the cloud-filtering of SIF retrievals. This is much more relaxed than for OCO $-2 \mathrm{XCO}_{2}$ retrievals, where data yields of only $7 \%$ are expected. The yields based on simulator data for the $100 \mathrm{hPa}, 50 \mathrm{hPa}$, and $10 \mathrm{hPa}$ thresholds are about $50 \%, 36 \%$, and $14 \%$, respectively.

\section{Vegetation indices from $0 \mathrm{CO}-2$}

One of the standard retrieval products from the OCO-2 are surface albedos and slopes within the 3 bands at $0.76,1.6$, and $2.0 \mu \mathrm{m}$. Owing to the high spectral resolution, these albedos are unperturbed by gaseous absorption and also implicitly corrected for atmospheric scattering by the full-physics 3-band OCO-2 retrieval algorithm (Bösch et al., 2006; O'Dell et al., 2012). In addition, cloud and aerosol filtering based on the strongly absorbing oxygen band as well as the $\mathrm{CO}_{2}$ ratio approach is very efficient and robust.

Vegetation water content indices (Fensholt \& Sandholt, 2003; Gao, 1996; Hunt \& Rock, 1989) are a good example of the extra information that we can obtain from the OCO-2 atmospherically corrected surface reflectance data, since the shortwave infrared (SWIR) domain $(1.4-3 \mu \mathrm{m})$ is strongly affected by liquid water absorption, whereas the near infrared (NIR) region $(0.75-1.4 \mu \mathrm{m})$ is relatively insensitive, a combination of both bands can be used to assess vegetation liquid water content. Following the formulation of the Normalized Difference Infrared Index (NDII) described by Fensholt and Sandholt (2003), we can write

$\mathrm{NDII}=\frac{R_{\mathrm{NIR}}-R_{\mathrm{SWIR}}}{R_{\mathrm{NIR}}+R_{\mathrm{SWIR}}}$

where $R$ refers to surface reflectance in the NIR and SWIR regions (MODIS channels at 0.86 and $1.64 \mu \mathrm{m}$ by Fensholt and Sandholt (2003), and 0.758 or $0.770 \mu \mathrm{m}$ and $1.6 \mu \mathrm{m}$ for OCO-2). Fig. 14 shows a temporal series from $06 / 2009$ to $01 / 2011$ of the retrieved NDII derived

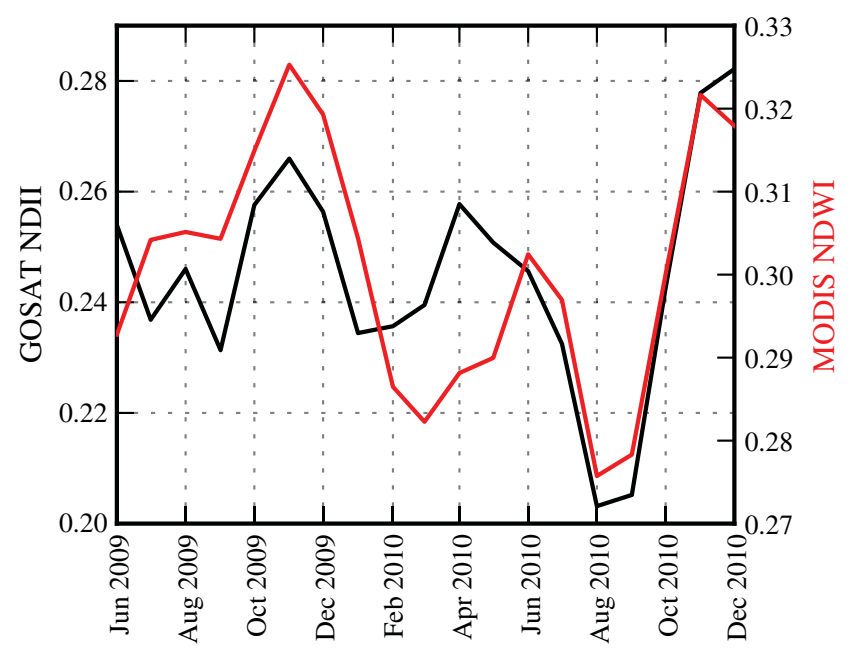

Fig. 14. NDII calculated with GOSAT-FTS data and NDWI from MODIS 06/2009-01/2011 for the Amazon region (lat: $-10^{\circ}$ to $2.5^{\circ}$, lon: $-75^{\circ}$ to $-55^{\circ}$ ). The fitted smooth curves are derived by using the smoothing spline method. The extreme drought in mid 2010 is clearly visible in both indices. 
from GOSAT-FTS data equivalent to OCO-2 measurements (NIR and SWIR at 0.758 and $1.55 \mu \mathrm{m}$, respectively) in the Amazon region (lat: $-10^{\circ}$ to $2.5^{\circ}$, lon: $-75^{\circ}$ to $-55^{\circ}$ ) together with the MODIS Normalized Difference Water Index (NDWI) (Gao, 1996) derived from MODIS reflectance data (NIR and SWIR at $860 \mathrm{~nm}$ and $1240 \mathrm{~nm}$, respectively). It can be observed that the NDII derived from the GOSAT-FTS shows a good agreement with the NDWI from the MODIS, which has been widely used in vegetation remote sensing applications (Fensholt \& Sandholt, 2003; Gao, 1996; Hunt \& Rock, 1989), in particular for the assessment of vegetation water stress. In this example, both indices are capturing the extreme 2010 drought event in the Western Amazon Basin (Lewis, Brando, Phillips, van der Heijden, \& Nepstad, 2011).

There are two other features of OCO-2 data of potential interest for vegetation remote sensing: one is the capability to provide very accurate reflectance and reflectance slope measurements at the longest spectral range of the vegetation red-edge window $(757 \mathrm{~nm})$, which can help to disentangle leaf biochemical constituents from canopy structure (Knyazikhin et al., 2012). The other is the smaller sensitivity of fluorescence to cloud and aerosol contamination than that of the reflectance-based data, which can be a critical improvement in tropical rainforest areas where vegetation indices face the greatest difficulties to provide reliable signals due to atmospheric effects (Hilker et al., 2012).

\section{Conclusions}

We have used the Thermal VACuum (TVAC) data from the actual OCO-2 instrument as well as orbit simulations for a full Nadir repeat cycle of 16 days to study the prospects of OCO-2 for remote sensing of chlorophyll fluorescence. We found that the instrument performs very well and will provide solar-induced chlorophyll fluorescence at $755 \mathrm{~nm}\left(\mathrm{SIF}_{755}\right)$ with a single-measurement precision of about $0.3-0.5 \mathrm{Wm}^{-2} \mathrm{sr}^{-1} \mu \mathrm{m}^{-1}$ and accuracy of better than $0.05 \mathrm{Wm}^{-2} \mathrm{sr}^{-1} \mu \mathrm{m}^{-1}$. While these numbers are already better than current retrievals from the GOSAT satellite, the biggest advancement will be achieved by the dramatic increase in measurement frequency (100 fold compared to GOSAT) and much smaller ground-pixel sizes $\left(1.3 \times 2.25 \mathrm{~km}^{2}\right)$. The OCO-2 will thus provide accurate near-global data for each repeat cycle (as opposed to a coarse seasonal average for GOSAT). The sun-synchronous orbit with 1:30 p.m. local overpass time will enable mid day SIF measurements similar to the GOSAT but with higher fidelity and much reduced standard error. The very narrow swath of OCO-2 won't allow for truly global retrievals as does GOME-2 (Joiner et al., 2013) but we found that this sampling bias will be small when averaged over regional (e.g. $1 \times 1^{\circ}$ ) scales, thus only hampering local studies either not covered by the OCO-2 swaths or with gradients of interest not properly aligned with the flight track.

We also studied the impact of scattering on the SIF retrievals on the global scale and found that cloud and aerosol filtering for the SIF retrievals can be much more relaxed compared to the strict filtering necessary for the $X_{\mathrm{CO}_{2}}$ retrievals. In fact, even cloudy pixels may be of interest for carbon cycle studies as relaxed cloud filters minimize a potential clear-sky bias in the SIF retrievals. Further studies are needed to quantify this trade-off between retrieval and sampling bias.

Eventually, consolidation of biophysics based fluorescence to GPP modeling (e.g. Van der Tol, Verhoef, \& Rosema, 2009; Van der Tol, Verhoef, Timmermans, et al., 2009) will be needed to fully exploit the potential of space-based SIF observations. We hope that OCO-2 will facilitate this as SIF validation along the narrow orbit and small footprints will actually become feasible for the first time.

\section{Acknowledgments}

Part of the research described in this paper was carried out by the Jet Propulsion Laboratory, California Institute of Technology, under a contract with the National Aeronautics and Space Administration. (C2013.
All rights reserved. The Colorado State University contributions to the ACOS task were supported by NASA contract 1439002 . We thank 4 anonymous reviewers for thorough and constructive reviews that strengthened the manuscript substantially.

\section{Appendix A}

\section{Details of the OCO-2 orbit simulator}

The OCO-2 orbit simulator is a modular code, developed at Colorado State University (CSU), to provide realistic modeling of reflected shortwave radiances as might be measured via satellite sensors at top of atmosphere (TOA). Descriptions of the mechanics pertaining to orbit paths, viewing geometries and other details that are not particularly relevant to the current research can be found in O'Brien, Polonsky, O'Dell, and Carheden (2009).

In short, the simulator can be set up to emulate a polar-orbiting satellite, including Nadir, Glint, and special Target viewing geometries with sampling frequency and FOV the same as that expected from the OCO-2. The successive order of interaction (SOI) radiative transfer solver (Heidinger, O'Dell, Bennartz, \& Greenwald, 2006; O'Dell, Heidinger, Greenwald, Bauer, \& Bennartz, 2006), coupled with the low-stream interpolator (LSI) (O'Dell, 2010) produces scalar radiances at the appropriate spectral bands and with the desired spectral resolution. Polarized radiances are determined via a second-order of scattering approximation (Natraj \& Spurr, 2007). An instrument model is provided based on best guess and TVAC testing of the OCO- 2 instrument and surface information is provided by the MODIS. For the current simulations, meteorological information was provided from the ECMWF reanalysis.

Of particular relevance to the current research is the modeling of clouds and aerosols in the simulations. Due to the rather cumbersome nature of the OCO-2 full physics retrieval algorithm, only approximately $6-7 \%$ of the total number of measured soundings will be inverted to yield $X_{\mathrm{CO}_{2}}$. Scenes heavily contaminated by cloud will be easily removed from processing via cloud screening, as described below. Therefore, simulations were performed using statistical sampling of a database containing optical thicknesses less than approximately 5.

The cloud and aerosol database in the current work is based on high vertical resolution profiles of optical thickness as measured by the Cloud-Aerosol Lidar with Orthogonal Polarization (CALIOP) sensor aboard the Cloud-Aerosol Lidar and Infrared Pathfinder Satellite Observation (CALIPSO) satellite in the A-train (Winker et al., 2007). This is the same orbit into which the OCO-2 is planned to be launched. For each time and location as the simulator stepped through an orbit, a single vertical CALIPSO profile was selected at random from a monthly database of $2^{\circ}$ by $2^{\circ}$ latitude/longitude boxes. To best represent the selected vertical CALIPSO profile, appropriate cloud and aerosol optical properties files, needed in the Mie radiative transfer calculations, were taken from a static internal database.

Cloud screening on the simulated data set was performed using both the Oxygen A-band retrieval ( $\mathrm{ABO} 2$ for short) that was detailed by (Taylor et al., 2012) and the IMAP-DOAS retrieval described by (Frankenberg, Platt, \& Wagner, 2005). The ABO2 relies primarily on the difference between the retrieved surface pressure $\left(\mathrm{p}_{\mathrm{s}}\right)$ and the estimated value from the ECMWF forecast. Truncation (lengthening) of the optical path due to reflection (multiple scattering) from clouds manifests as underestimates (overestimates) of $\mathrm{p}_{\mathrm{s}}$. Previous work has demonstrated that the $\mathrm{ABO} 2$ cloud screen alone can remove scenes contaminated with optical thicknesses greater than approximately $0.3 \mathrm{OD}$, except for those with clouds at low altitudes, where path length modification is too small relative to the total path (O'Dell et al., 2012).

The IMAP-DOAS routine utilizes ratios in retrieved $\mathrm{CO}_{2}$ between the weak and strong absorption bands, and similar ratios of $\mathrm{H}_{2} \mathrm{O}$ to distinguish clear from cloudy scenes. Unpublished work has shown that the IMAP-DOAS routine is efficient at removing low, thick clouds, thereby making the combination of the two cloud screeners very powerful. 
The current OCO- 2 operations call for these two algorithms to be run on the entire OCO- 2 data set $(\simeq 1$ million soundings per day), the results of which will be passed to a scene selection algorithm to determine which scenes will be processed by the computationally expensive full physics retrieval code. The SIF retrievals, however, will be part of the preprocessor and as such not be subject to pre-filtering.

\section{References}

Baker, N. (2008). Chlorophyll fluorescence: A probe of photosynthesis in vivo. Annual Review of Plant Biology, 59, 89.

Beer, C., Reichstein, M., Tomelleri, E., Ciais, P., Jung, M., Carvalhais, N., et al. (2010). Terrestrial gross carbon dioxide uptake: Global distribution and covariation with climate. Science, 329, 834.

Behrenfeld, M. J., \& Milligan, A. J. (2013). Photophysiological expressions of iron stress in phytoplankton. Annual Review of Marine Science, 5, 217-246. http://dx.doi.org/10. 1146/annurev-marine-121211-172356 (pMID: 22881354.).

Bösch, H., Toon, G., Sen, B., Washenfelder, R., Wennberg, P., Buchwitz, M., et al. (2006). Space-based near-infrared $\mathrm{CO}_{2}$ measurements: Testing the Orbiting Carbon Observatory retrieval algorithm and validation concept using SCIAMACHY observations over Park Falls, Wisconsin. Journal of Geophysical Research, 111, 0148-0227.

Campbell, P., Middleton, E., Corp, L. A., \& Kim, M. S. (2008). Contribution of chlorophyll fluorescence to the apparent vegetation reflectance. Science of the Total Environment, 404, 433-439.

Corp, L., Middleton, E., McMurtrey, J., Entcheva Campbell, P., \& Butcher, L. (2006). Fluorescence sensing techniques for vegetation assessment. Applied Optics, 45, 1023-1033.

Fensholt, R., \& Sandholt, I. (2003). Derivation of a shortwave infrared water stress index from MODIS near- and shortwave infrared data in a semiarid environment. Remote Sensing of Environment, 87, 111-121. http://dx.doi.org/10.1016/j.rse.2003.07.002 (http://www.sciencedirect.com/science/article/pii/S0034425703001895)

Frankenberg, C., Butz, A., \& Toon, G. C. (2011). Disentangling chlorophyll fluorescence from atmospheric scattering effects in O-2 A-band spectra of reflected sun-light. Geophysical Research Letters, 38, L03801.

Frankenberg, C., Fisher, J., Worden, J., Badgley, G., Saatchi, S., Lee, J. E., et al. (2011). New global observations of the terrestrial carbon cycle from GOSAT: Patterns of plant fluorescence with gross primary productivity. Geophysical Research Letters, 38, L17706.

Frankenberg, C., O'Dell, C., Guanter, L., \& McDuffie, J. (2012). Remote sensing of nearinfrared chlorophyll fluorescence from space in scattering atmospheres: implications for its retrieval and interferences with atmospheric $\mathrm{CO}_{2}$ retrievals. Atmospheric Measurement Techniques, 5, 2081-2094.

Frankenberg, C., Platt, U., \& Wagner, T. (2005). Iterative maximum a posteriori (IMAP)-DOAS for retrieval of strongly absorbing trace gases: Model studies for $\mathrm{CH}_{4}$ and $\mathrm{CO}_{2}$ retrieval from near infrared spectra of SCIAMACHY onboard ENVISAT. Atmospheric Chemistry and Physics, 5, 9-22. http://dx.doi.org/10.5194/acp-5-9-2005 (URL: http://www. atmos-chem-phys.net/5/9/2005/)

Gao, B. C. (1996). "NDWI"-A normalized difference water index for remote sensing of vegetation liquid water from space. Remote Sensing of Environment, 58, 257-266. http://dx.doi.org/10.1016/S0034-4257(96)00067-3.

Genty, B., Briantais, J., \& Baker, N. (1989). The relationship between the quantum yield of photosynthetic electron transport and quenching of chlorophyll fluorescence. Biochimica et Biophysica Acta, 990, 87-92.

Gu, L., Baldocchi, D.D., Verma, S. B., Black, T. A., Vesala, T., Falge, E. M., et al. (2002). Advantages of diffuse radiation for terrestrial ecosystem productivity. Journal of Geophysical Research, 107. http://dx.doi.org/10.1029/2001JD001242.

Gu, L., Baldocchi, D.D., Wofsy, S.C., Munger, J. W., Michalsky, J. J., Urbanski, S. P., et al (2003). Response of a deciduous forest to the Mount Pinatubo eruption: Enhanced photosynthesis. Science, 299, 2035-2038.

Guanter, L., Dudhia, A., Lewis, P. E., Gómez-Dans, J., Kuze, A., Suto, H., et al. (2012). Retrieval and global assessment of terrestrial chlorophyll fluorescence from GOSAT space measurements. Remote Sensing of Environment, 121, 236-251.

Guanter, L., Rossini, M., Colombo, R., Meroni, M., Frankenberg, C., Lee, J. E., et al. (2013). Using field spectroscopy to assess the potential of statistical approaches for the retrieval of sun-induced chlorophyll fluorescence from ground and space. Remote Sensing of Environment, 133, 52-61.

Hamazaki, T., Kaneko, Y., Kuze, A., \& Kondo, K. (2005). Fourier transform spectrometer for greenhouse gases observing satellite (GOSAT). Proceedings of SPIE (pp. 73).

Heidinger, A. K., O'Dell, C., Bennartz, R., \& Greenwald, T. (2006). The successive-order-ofinteraction radiative transfer model: Part I: Model development. Journal of Applied Mathematics and Computing, 45, 1388-1402. http://dx.doi.org/10.1175/JAM2387.1.

Hilker, T., Lyapustin, A. I., Tucker, C. J., Sellers, P. J., Hall, F. G., \& Wang, Y. (2012). Remote sensing of tropical ecosystems: Atmospheric correction and cloud masking matter. Remote Sensing of Environment, 127, 370-384. http://dx.doi.org/10.1016/j.rse.2012.08.03.

Hunt, E. Raymond, \& Rock, Barrett N. (1989). Detection of changes in leaf water content using near- and middle-infrared reflectances. Remote Sensing of Environment, 30, 43-54. http://dx.doi.org/10.1016/0034-4257(89)90046-1.
Joiner, J., Guanter, L., Lindstrot, R., Voigt, M., Vasilkov, A. P., Middleton, E. M., et al. (2013). Global monitoring of terrestrial chlorophyll fluorescence from moderate spectral resolution near-infrared satellite measurements: methodology, simulations, and application to GOME-2. Atmospheric Measurement Techniques Discussions, 6, 3883-3930. http://dx.doi.org/10.5194/amtd-6-3883-2013.

Joiner, J., Yoshida, Y., Vasilkov, A. P., Middleton, E. M., Campbell, P. K. E., Yoshida, Y., et al. (2012). Filling-in of near-infrared solar lines by terrestrial fluorescence and other geophysical effects: Simulations and space-based observations from SCIAMACHY and GOSAT. Atm0spheric Measurement Techniques, 5, 809-829.

Joiner, J., Yoshida, Y., Vasilkov, A. P., Yoshida, Y., Corp, L. A., \& Middleton, E. M. (2011). First observations of global and seasonal terrestrial chlorophyll fluorescence from space. Biogeosciences, 8, 637-651. http://dx.doi.org/10.5194/bg-8-637-2011 (URL: http://www.biogeosciences.net/8/637/2011/)

Kanniah, K. D., Beringer, J., North, P., \& Hutley, L. (2012). Control of atmospheric particles on diffuse radiation and terrestrial plant productivity: A review. Progress in Physical Geography, 36, 209-237. http://dx.doi.org/10.1177/0309133311434244.

Knyazikhin, Y., Schull, M.A., Stenberg, P., Mõttus, M., Rautiainen, M., Yang, Y., et al. (2012). Hyperspectral remote sensing of foliar nitrogen content. Proceedings of the National Academy of Sciences. http://dx.doi.org/10.1073/pnas.1210196109.

Krause, G. H., \& Weis, E. (1984). Chlorophyll fluorescence as a tool in plant physiology. Photosynthesis Research, 5, 139-157.

Kuze, A., Suto, H., Nakajima, M., \& Hamazaki, T. (2009). Thermal and near infrared sensor for carbon observation Fourier-transform spectrometer on the Greenhouse Gases Observing Satellite for greenhouse gases monitoring. Applied Optics, 48, 6716-6733.

Lee, J. E., Frankenberg, C., van der Tol, C., Berry, J. A., Guanter, L., Boyce, C. K., et al. (2013). Forest productivity and water stress in Amazonia: Observations from GOSAT chlorophyll fluorescence. Proceedings of the Royal Society B: Biological Sciences, 280. http://dx.doi.org/10.1098/rspb.2013.0171.

Lewis, S. L., Brando, P.M., Phillips, O. L., van der Heijden, G. M. F., \& Nepstad, D. (2011). The 2010 Amazon drought. Science, 331, 554. http://dx.doi.org/10.1126/science.1200807.

Moya, I., Camenen, L., Evain, S., Goulas, Y., Cerovic, Z., Latouche, G., et al. (2004). A new instrument for passive remote sensing: 1. Measurements of sunlight-induced chlorophyll fluorescence. Remote Sensing of Environment, 91, 186-197.

Natraj, V., \& Spurr, R. J. (2007). A fast linearized pseudo-spherical two orders of scattering model to account for polarization in vertically inhomogeneous scattering absorbing media. Journal of Quantitative Spectroscopy and Radiative Transfer, 107, 263-293. http://dx.doi.org/10.1016/j.jqsrt.2007.02.01 (URL: http://www.sciencedirect.com/ science/article/pii/S0022407307001124)

Neville, R. A., \& Gower, J. F. R. (1977). Passive remote sensing of phytoplankton via chlorophyll $\alpha$ fluorescence. Journal of Geophysical Research, 82, 3487-3493. http://dx.doi. org/10.1029/JC082i024p03487.

O'Brien, D.M., Polonsky, I., O'Dell, C., \& Carheden, A. (2009). Orbiting Carbon Observatory (OCO), algorithm theoretical basis document: The OCO simulator. Technical Report. Cooperative Institute for Research in the Atmosphere.

O'Dell, C. W. (2010). Acceleration of multiple-scattering, hyperspectral radiative transfer calculations via low-streams interpolation. Journal of Geophysical Research, 115. http://dx.doi.org/10.1029/2009JD012803.

O'Dell, C. W., Connor, B., Bösch, H., O'Brien, D., Frankenberg, C., Castano, R., et al. (2012). The $A \operatorname{COS} \mathrm{CO}_{2}$ retrieval algorithm-Part 1: Description and validation against synthetic observations. Atmospheric Measurement Techniques, 5, 99-121. http://dx.doi.org/10. 5194/amt-5-99-2012.

O'Dell, C., Heidinger, A. K., Greenwald, T., Bauer, P., \& Bennartz, R. (2006). The successiveorder-of-interaction radiative transfer model: Part II: Model performance and applications. Journal of Applied Mathematics and Computing, 45, 1403-1413. http://dx.doi. org/10.1175/JAM2409.1.

Parazoo, N. C., Bowman, K., Frankenberg, C., Lee, J. E., Fisher, J. B., Worden, J., et al. (2013). Interpreting seasonal changes in the carbon balance of southern Amazonia using measurements of $\mathrm{XCO}_{2}$ and chlorophyll fluorescence from GOSAT. Geophysical Research Letters, 40, 2829-2833. http://dx.doi.org/10.1002/grl.50452.

Taylor, T. E., O'Dell, C., O'Brien, D., Kikuchi, N., Yokota, T., Nakajima, T., et al. (2012). Comparison of cloud-screening methods applied to GOSAT near-infrared spectra. IEEE Transactions on Geoscience and Remote Sensing, 50, 295-309. http://dx.doi.org/10. 1109/TGRS.2011.2160270.

Van der Tol, C., Verhoef, W., \& Rosema, A. (2009). A model for chlorophyll fluorescence and photosynthesis at leaf scale. Agricultural and forest meteorology, 149, 96-105.

Van der Tol, C., Verhoef, W., Timmermans, J., Verhoef, A., \& Su, Z. (2009). An integrated model of soil-canopy spectral radiance observations, photosynthesis, fluorescence, temperature and energy balance. Biogeosciences, 6, 3109-3129.

Winker, D.M., Hunt, W. H., \& McGill, M. J. (2007). Initial performance assessment of CALIOP. Geophysical Research Letters, 34, L19803. http://dx.doi.org/10.1029/2007GL030135.

Xing, X. G., Zhao, D. Z., Liu, Y. G., Yang, J. H., Xiu, P., \& Wang, L. (2007). An overview of remote sensing of chlorophyll fluorescence. Ocean Science Journal, 42, 49-59. http://dx. doi.org/10.1007/BF03020910. 